\title{
Reentry Trajectory Optimization for Hypersonic Vehicles Using Fuzzy Satisfactory Goal Programming Method
}

\author{
Chao-Fang $\mathrm{Hu} \quad$ Yue Xin \\ School of Electrical Engineering and Automation, Tianjin University, Tianjin 300072, China
}

\begin{abstract}
Constrained reentry trajectory optimization for hypersonic vehicles is a challenging job. In particular, this problem becomes more difficult when several objectives with preemptive priorities are expected for different purposes. In this paper, a fuzzy satisfactory goal programming method is proposed to solve the multi-objective reentry trajectory optimization problem. Firstly, direct collocation approach is used to discretize the reentry trajectory optimal-control problem with nonlinear constraints into nonlinear multiobjective programming problem with preemptive priorities, where attack angles and bank angles at nodes and collocation nodes are selected as control variables. Secondly, the preemptive priorities are transformed into the relaxed order of satisfactory degrees according to the principle that the objective with higher priority has higher satisfactory degree. Then the fuzzy satisfactory goal programming model is proposed. The balance between optimization and priorities is realized by regulating parameter $\lambda$, such that the satisfactory reentry trajectory can be acquired. The simulation demonstrates that the proposed method is effective for the multi-objective reentry trajectory optimization of hypersonic vehicles.
\end{abstract}

Keywords: Reentry trajectory optimization, direct collocation method, multi-objective optimization, fuzzy satisfactory method, goal programming.

\section{Introduction}

The aircrafts which have the velocity of Mach 5 or more are defined as hypersonic vehicles. Obviously, hypersonic vehicles will play an increasingly important role in the future. Therefore, a well-designed trajectory, particularly in reentry phase, is key to the stable flight of hypersonic vehicles $^{[1]}$.

The trajectory optimization methods are categorized into two major types, i.e., direct and indirect methods ${ }^{[2]}$. Most early investigators adopt the latter ${ }^{[3]}$. It is necessary for the indirect methods to derive the optimality condition called Hamiltonian boundary value. However, the radius of convergence is so small that acquiring this condition is very difficult. Thus, the direct approaches are applied frequently, such as direct shooting method ${ }^{[4]}$, pseudospectral $\operatorname{method}^{[5]}$, direct collocation method ${ }^{[6]}$, etc. For example, $\mathrm{Qu}^{[4]}$ uses multiple shooting technique to calculate the reentry trajectory of American shuttle orbit. Huntington and $\mathrm{RaO}^{[7]}$ design the gauss pseudospectral method for the optimal control of spacecraft. Bibeau ${ }^{[8]}$ adopt the collocation method to design the optimal reentry trajectory of a fixedtrim vehicle.

Generally, the traditional design of reentry trajectory usually aims at a single objective, such as maximizing the range, maximizing the terminal velocity, etc. However, the

\footnotetext{
Regular paper

Manuscript received September 27, 2013; accepted January 21, 2014 This work was supported by National Natural Science Foundation of China (No.61074064) and Natural Science Foundation of Tianjin (No. 12JCZDJC30300).

Recommended by Associate Editor Jyh-Horng Chou

(C)Institute of Automation, Chinese Academy of Science and Springer-Verlag Berlin Heidelberg 2015
}

expectations for reducing costs and enhancing performance are increasing gradually; this brings about the development of multi-objective optimization (MOO) for reentry trajectory. In early times, the method based on weighting factors is widely used to transform multi-objective problem into traditional single objective formulation ${ }^{[9]}$. But it is difficult to determine the weights. In addition, Chen et al. ${ }^{[10]}$ apply NSGA-II algorithm to realize a two-objective optimization design of reentry trajectory, however, the computation burden of iterative optimization is heavy and the importance of different objectives cannot be reflected directly. Then Yong et al. ${ }^{[1]}$ use the physical programming (PP) method to determine gliding reentry trajectory. Nevertheless, selecting the preference function is still difficult, and the formulated programming model is strongly nonlinear.

Preemptive priorities, as a special case of importance of objectives, require all the objectives to be solved in sequence. For this requirement, different methods have been attempted, such as lexicographic optimization ${ }^{[12]}$, varyingdomain optimization ${ }^{[13]}$, and two-step optimization ${ }^{[14]}$. For MOO, goal programming (GP), initially introduced by Charnes and Cooper ${ }^{[15]}$, is also a promising methodology. It considers all the objectives with different attainment relations through minimizing the deviations from the expected values, which makes it easy to reformulate MOO model with priorities. Accordingly, a fuzzy satisfactory goal programming (FSGP) method is proposed in this paper. Firstly, direct collocation method is adopted to discretize the dynamic model and constraints of hypersonic vehicles by selecting attack angle and bank angle at each node and collocation node as control variables. Then the dynamic optimal control problem is transformed into a static nonlinear 
multi-objective programming problem with preemptive priorities. Secondly, all the objectives are fuzzified using membership functions. Their preemptive priorities are modelled into a relaxed order of satisfactory degrees according to the principle that the objective with higher priority has higher satisfactory degree ${ }^{[16]}$. Thus, the FSGP model for three fuzzy relations is designed. By adjusting the parameter, the optimal reentry trajectory can be generated. It not only realizes optimization of all the objectives, but also satisfies the requirement of preemptive priorities. Finally, the simulation demonstrates our method is effective for multiobjective reentry trajectory optimization of hypersonic vehicles.

Section 2 describes reentry trajectory optimization problem of hypersonic vehicles. The FSGP method is proposed in Section 3. Section 4 demonstrates its power by simulation. In Section 5 the conclusions are drawn.

\section{Problem formulation}

\subsection{Reentry dynamics of hypersonic vehi- cles}

The following assumptions are presented:

1) The earth is a uniform sphere.

2) The earth is flat, and its revolution is ignored.

3) The reentry vehicle is considered as a point.

4) The sideslip angle is kept to zero.

Then, the equations of motion are given as follows ${ }^{[17]}$ :

$$
\begin{aligned}
\dot{r} & =v \sin \gamma \\
\dot{\phi} & =\frac{v \cos \gamma \sin \chi}{r \cos \theta} \\
\dot{\theta} & =\frac{v \cos \gamma \cos \chi}{r} \\
\dot{v} & =-\frac{D}{m}-g \sin \gamma \\
\dot{\chi} & =\frac{L \sin \sigma}{m v \cos \gamma}+\frac{v}{r} \cos \gamma \sin \chi \tan \theta \\
\dot{\gamma} & =\frac{L \cos \sigma}{m v}-\frac{g \cos \gamma}{v}+\frac{v \cos \gamma}{r}
\end{aligned}
$$

where $r, \phi, \theta, v, \chi, \gamma$ are state variables, representing radial position, latitude, longitude, velocity, heading angle, and flight-path angle respectively. Attack angle $\alpha$ and bank angle $\sigma$ are control variables. $g=\frac{\mu}{r^{2}}$ is gravity, and $\mu=1.4076539 \times 10^{16} \mathrm{ft}^{3} / \mathrm{s}^{2}$ is gravitational parameter of the earth; $m$ is mass of the vehicle. $L$ and $D$ are lift and drag forces respectively which are defined as

$$
L=\frac{1}{2} \rho v^{2} C_{L} S, \quad D=\frac{1}{2} \rho v^{2} C_{D} S
$$

where $S$ is reference area of the vehicle. $\rho$ represents atmospheric density, and it is formulated as the following exponential atmosphere model $^{[17]}$

$$
\rho=\rho_{0} \mathrm{e}^{-k\left(r-R_{0}\right)}
$$

where $\rho_{0}=0.002378 \mathrm{slug} / \mathrm{ft}^{3}, R_{0}=20902900 \mathrm{ft}$ is radius of the earth, and $k=4.20168 \times 10^{-5} / \mathrm{ft}$. $C_{L}$ and $C_{D}$ are lift and drag coefficients ${ }^{[17]}$

$$
\begin{aligned}
& C_{L}=C_{L 0}+C_{L 1} \alpha \\
& C_{D}=C_{D 0}+C_{D 1} \alpha+C_{D 2} \alpha^{2}
\end{aligned}
$$

where $C_{L 0}=-0.2070, C_{L 1}=1.676, C_{D 0}=0.07854, C_{D 1}=$ $-0.3529, C_{D 2}=2.040$.

\subsection{Constraints}

In reentry process, the hypersonic vehicle should satisfy some constraints due to safety and stability, as well as the requirement of its structure and material. These constraints mainly contain initial and terminal constraints, path constraints and boundary constraints.

\subsubsection{Initial and terminal constraints}

Initial and terminal constraints specify the requirements for initial and final states of reentry process. In this paper, initial constraints about all the states are defined as

$$
[r, \phi, \theta, v, \chi, \gamma]=\left[r_{0}, \phi_{0}, \theta_{0}, v_{0}, \chi_{0}, \gamma_{0}\right]
$$

and terminal constraints about $r, \gamma, v$ are written as

$$
[r, \gamma, v]=\left[r_{f}, \gamma_{f}, v_{f}\right]
$$

The subscripts " 0 " and " $f$ " represent initial and final time, respectively.

\subsubsection{Path constraints}

In order to prevent the temperature of the hypersonic vehicle surface from being too high, heat flux at the stagnation point should be limited. This is useful to reduce the weight of heat-resistant material and the cost. Therefore the heat flux constraint is defined as

$$
\dot{Q}=\left(h_{0}+h_{1} \alpha+h_{2} \alpha^{2}+h_{3} \alpha^{3}\right) \cdot C \rho^{0.5} v^{3.07} \leq \dot{Q}_{\max }
$$

where $C=9.289 \times 10^{-9} \mathrm{Btu} \cdot \mathrm{s}^{2.07} / \mathrm{ft}^{3.57} / \mathrm{slug}^{0.5}, h_{0}=$ 1.067, $h_{1}=-1.101, h_{2}=0.6988, h_{3}=-0.1903$.

In addition, it is necessary to restrict dynamic pressure in order to protect the structure of the vehicle and realize attitude control. Thus constraint of dynamic pressure $q$ is formulated as

$$
q=\frac{1}{2} \rho v^{2} \leq q_{\max } .
$$

To ensure flight safety, overload $n$ is also subjected to the following constraint

$$
n=\frac{\sqrt{L^{2}+D^{2}}}{m g} \leq n_{\max } .
$$

\subsubsection{Boundary constraints}

For the hypersonic vehicle, the state variables should be limited as

$$
\begin{array}{ll}
r_{\min } \leq r \leq r_{\max } & \theta_{\min } \leq \theta \leq \theta_{\max } \\
\phi_{\min } \leq \phi \leq \phi_{\max } & v_{\min } \leq v \leq v_{\max } \\
\chi_{\min } \leq \chi \leq \chi_{\max } & \gamma_{\min } \leq \gamma \leq \gamma_{\max }
\end{array}
$$

and the boundaries of the control variables are given as

$$
\alpha_{\min } \leq \alpha \leq \alpha_{\max } \quad \sigma_{\min } \leq \sigma \leq \sigma_{\min }
$$




\subsection{Optimization objectives}

The maximum cross range, i.e., terminal longitude, is an important indicator to measure the reentry flying ability of hypersonic vehicles. Moreover, aerodynamic heating is very serious and is also a huge challenge to structural design in the reentry process. Besides, severe oscillation is not desired for better stability, and a larger terminal velocity will also provide more kinetic energy for the vehicle. Thus, the following four objectives should be achieved.

1) Maximizing the cross range:

$$
\max f_{1}=\theta\left(t_{f}\right)
$$

2) Minimizing the total aerodynamic heating:

$$
\min f_{2}=\int_{t_{0}}^{t_{f}} \dot{Q}(t) \mathrm{d} t
$$

3) Minimizing the oscillation:

$$
\min f_{3}=\int_{t_{0}}^{t_{f}} \dot{\gamma}^{2}(t) \mathrm{d} t
$$

4) Maximizing the terminal velocity:

$$
\max f_{4}=u\left(t_{f}\right) .
$$

\subsection{Preemptive priorities of objectives}

Considering the promising prospects of hypersonic vehicles in combat and reconnaissance, the primary task is to achieve a larger flight range and a higher terminal velocity, which can make the vehicle have larger striking range and capability. Therefore, the two objectives, "a) maximizing the cross range" and "d) maximizing the terminal velocity" are selected as the first level. Then, reducing aerodynamic heating and oscillation are considered, where the objective "b) minimizing the total aerodynamic heating" is selected as the second level, and the objective "c) minimizing the oscillation" is the third level. That is

Level one: a) and d);

Level two: b);

Level three: c).

Combining the above constraints, objectives and preemptive priorities, the multi-objective reentry trajectory optimization model of hypersonic vehicles can be formulated as

$$
\left\{\begin{array}{cc}
\min & {\left[P_{1}\left(-f_{1}(x, u),-f_{4}(x, u)\right)\right.} \\
& \left.P_{2}\left(f_{2}(x, u)\right), P_{3}\left(f_{3}(x, u)\right)\right] \\
\text { s.t. } & (x, u) \in G
\end{array}\right.
$$

where $x, u$ refer to state variables and control variables, i.e., $x=(r, \phi, \theta, v, \chi, \gamma) \in \mathbf{R}^{6}$, and $u=(\alpha, \sigma) \in \mathbf{R}^{2} ; P_{i}$, $i=1,2,3$ represents priority factors of the objectives, and $P_{i}>>P_{i+1}$. This means the objectives belonging to $P_{i}$ have higher priority than those of $P_{i+1} ; G$ is the intersection of constraints (11)-(17).

\section{Reentry trajectory optimization based on FSGP}

Since the MOO model (22) consists of differential motion equations (1)-(6), its solution is not obtained by general algorithm directly. Firstly, direct collocation method is adopted to transform the differential constraints into algebraic formulations, which transform continuous optimalcontrol problem into discrete nonlinear multi-objective programming problem with preemptive priorities. Then, the objectives are fuzzified using different types of membership functions. The relaxed order of satisfactory degrees is used to model the preemptive priorities. By introducing GP, the FSGP model for three fuzzy relations is designed to find the satisfactory solution.

\subsection{Direct collocation method}

In direct collocation method ${ }^{[8,18]}$, state variables and control variables are discretized firstly. The motion equations of hypersonic vehicles are approximated by GaussLobatto polynomials. Then the motion equation constraints are transformed into a set of algebraic constraints. Thus, the reentry trajectory optimization problem is converted to a nonlinear programming problem.

In direct collocation method, different orders of polynomial can be chosen to approximate the dynamics. In this paper, the third order Simpson method is adopted.

\subsubsection{Discretization of time interval}

Divide the whole time process $\left[t_{0}, t_{f}\right]$ into $N$ segments which are expressed by $\left[t_{i-1}, t_{i}\right], i=1,2, \cdots, N$. The length of each segment is defined as $h_{i}=t_{i}-t_{i-1}$. Let $s=\frac{\left(t-t_{i-1}\right)}{h_{i}}$, then $s \in[0,1]$. The elements of the state variables $x$ are defined as $x_{1}=r, x_{2}=\phi, x_{3}=\theta, x_{4}=v, x_{5}=\chi$, and $x_{6}=\gamma$. Similarly, those of the control variables $u$ are $u_{1}=$ $\alpha$ and $u_{2}=\sigma$. Select the boundary points of each segment as nodes, then the state variables and control variables at nodes are expressed as $\left(x_{j}^{0}, x_{j}^{1}, \cdots, x_{j}^{N}\right), j=1,2, \cdots, 6$ and $\left(u_{k}^{0}, u_{k}^{1}, \cdots, u_{k}^{N}\right), k=1,2$ respectively.

\subsubsection{Approximation of state variables}

The state variables are approximated by the third order Hermite polynomials on each segment

$$
x=c_{0}+c_{1} s+c_{2} s^{2}+c_{3} s^{3} .
$$

The boundaries are

$$
\begin{array}{ll}
x^{i-1}=x(0), & x^{i}=x(1), \\
\dot{x}^{i-1}=\left.\frac{\mathrm{d} x}{\mathrm{~d} s}\right|_{s=0}, & \dot{x}^{i}=\left.\frac{\mathrm{d} x}{\mathrm{~d} s}\right|_{s=1} .
\end{array}
$$

By solving (24), the following equations can be achieved

$$
\begin{aligned}
& c_{0}=x^{i-1} \\
& c_{1}=\dot{x}^{i-1} \\
& c_{2}=-3 x^{i-1}-2 \dot{x}^{i-1}+3 x^{i}-\dot{x}^{i} \\
& c_{3}=2 x^{i-1}+\dot{x}^{i-1}-2 x^{i}+\dot{x}^{i} .
\end{aligned}
$$


Choose the midpoints of each time segment as collocation points, and set $s=\frac{1}{2}$. Then substituting (25) into (23), we can acquire

$$
\begin{aligned}
& x^{c i}=\frac{x^{i}+x^{i-1}}{2}+\frac{h_{i}\left(d^{i-1}-d^{i}\right)}{8} \\
& \dot{x}^{c i}=\frac{3\left(x^{i}-x^{i-1}\right)}{2 h_{i}}-\frac{\left(d^{i-1}+d^{i}\right)}{4}
\end{aligned}
$$

where the superscript " $c$ " represents collocation points; $d$ is the derivative of state variable at the nodes, which is derived from the motion equations.

\subsubsection{Calculation of defect vectors}

Integrating the motion equations by Simpson integral formula in each interval, we can obtain the defect vector

$\vec{D}=x^{i-1}-x^{i}+\frac{h_{i}}{6}\left[\mathrm{~d}\left(x^{i-1}, u^{i-1}\right)+4 \mathrm{~d}\left(x^{c i}, u^{c i}\right)+\mathrm{d}\left(x^{i}, u^{i}\right)\right]$

where $\mathrm{d}\left(x^{c i}, u^{c i}\right)$ is the derivative of state variable derived from motion equations evaluated at the collocation points.

In order to make equation (23) meet the change of state variables, the third order Simpson method requires the derivatives of state variables derived from polynomials and motion equations are equal, which can be achieved by letting Defect vector be equal to zero.

By the above approach, the motion equation constraints have been converted to algebraic constraints. Combining the constraints mentioned in Section 2.2, objectives mentioned in Section 2.3, and priorities in Section 2.4, the trajectory optimization problem is transformed into a nonlinear programming problem with the state variables and control variables at nodes and control variables at collocations being optimization variables.

\subsection{FSGP method}

For multiple objectives, an optimal solution meeting all of them simultaneously can be hardly found because of conflicts. Especially, it becomes more difficult when the preemptive priorities to divide the objectives into different levels are required.

Due to the difficulty of quantifying the optimization degree and priorities of objectives, we introduce fuzzy optimization, and use the concept of satisfactory degree to express optimization extent.

\subsubsection{Fuzzy goals}

Generally, different fuzzy goals can be defined for the objectives. This formulates fuzzy decision often presented as $^{[12]}$

$$
\left\{\begin{array}{cc}
\text { Find } & (x, u) \\
\text { s.t. } & f_{i}(x, u) \rightarrow f_{i}^{*}, i=1, \cdots, m, \quad(x, u) \in G
\end{array}\right.
$$

where $f_{i}(x, u)$ is the $i$-th objective function; $f_{i}^{*}$ is the perspective goal value for $f_{i}(x, u)$; " $\rightarrow$ " represents different fuzzy relations containing " $\leq$ ", " $\tilde{\geq}$ " and " $\tilde{=}$ ". Then we can define the membership functions for the three fuzzy relations using triangle-like membership functions.
The fuzzy relation " $\tilde{\leq}$ " represents the requirement of fuzzy objective being less than or equal to the expected value approximately. Its membership function is ${ }^{[19]}$ :

$\mu_{f_{i}}(x, u)=\left\{\begin{array}{cc}1, & f_{i}(x, u) \leq f_{i}^{*} \\ 1-\frac{f_{i}(x, u)-f_{i}^{*}}{f_{i}^{\max }-f_{i}^{*}}, & f_{i}^{*} \leq f_{i}(x, u) \leq f_{i}^{\max } \\ 0, & f_{i}(x, u) \geq f_{i}^{\max }\end{array}\right.$

with the tolerance interval of $\left(f_{i}^{*}, f_{i}^{\max }\right)$.

The membership function of " $\tilde{\geq}$ " is

$$
\mu_{f_{i}}(x, u)=\left\{\begin{array}{cc}
1, & f_{i}(x, u) \geq f_{i}^{*} \\
1-\frac{f_{i}^{*}-f_{i}(x, u)}{f_{i}^{*}-f_{i}^{\min }}, & f_{i}^{\min } \leq f_{i}(x, u) \leq f_{i}^{*} \\
0, & f_{i}(x, u) \leq f_{i}^{\text {min }}
\end{array}\right.
$$

where $\left(f_{i}^{\mathrm{min}}, f_{i}^{*}\right)$ is the tolerance interval which means that the objective is approximately more than or equal to its desired value.

Similarly, the relation " $\tilde{=}$ " denotes that the objective is in the vicinity of expectation. The tolerance interval is $\left(f_{i}^{\min }, f_{i}^{\max }\right)$. Its membership function is

$$
\mu_{f_{i}}(x, u)=\left\{\begin{array}{cc}
0, & f_{i}(x, u) \geq f_{i}^{\max } \\
1-\frac{f_{i}(x, u)-f_{i}^{*}}{f_{i}^{\max }-f_{i}^{*}}, & f_{i}^{*} \leq f_{i}(x, u) \leq f_{i}^{\max } \\
1, & f_{i}(x, u)=f_{i}^{*} \\
1-\frac{f_{i}^{*}-f_{i}(x, u)}{f_{i}^{*}-f_{i}^{\min },} & f_{i}^{\min } \leq f_{i}(x, u) \leq f_{i}^{*} \\
0, & f_{i}(x, u) \leq f_{i}^{\min } .
\end{array}\right.
$$

For the membership functions established above, the perspective goal value $f_{i}^{*}$ can be computed by single objective optimization. The lower and upper tolerance limits $f_{i}^{\text {min }}$ and $f_{i}^{\max }$ can be determined using the following payoff table constructed by optimal solution of single objective optimization (Table 1).

Table 1 Payoff table

\begin{tabular}{cccccc}
\hline & $f_{1}$ & $f_{2}$ & $f_{3}$ & $\cdots$ & $f_{m}$ \\
\hline Opt $f_{1}(x, u)$ & $f_{1}\left(x_{1}^{*}, u_{1}^{*}\right)$ & $f_{2}\left(x_{1}^{*}, u_{1}^{*}\right)$ & $f_{3}\left(x_{1}^{*}, u_{1}^{*}\right)$ & $\cdots$ & $f_{m}\left(x_{1}^{*}, u_{1}^{*}\right)$ \\
Opt $f_{2}(x, u)$ & $f_{1}\left(x_{2}^{*}, u_{2}^{*}\right)$ & $f_{2}\left(x_{2}^{*}, u_{2}^{*}\right)$ & $f_{3}\left(x_{2}^{*}, u_{2}^{*}\right)$ & $\cdots$ & $f_{m}\left(x_{2}^{*}, u_{2}^{*}\right)$ \\
Opt $f_{3}(x, u)$ & $f_{1}\left(x_{3}^{*}, u_{3}^{*}\right)$ & $f_{2}\left(x_{3}^{*}, u_{3}^{*}\right)$ & $f_{3}\left(x_{3}^{*}, u_{3}^{*}\right)$ & $\cdots$ & $f_{m}\left(x_{3}^{*}, u_{3}^{*}\right)$ \\
$\vdots$ & $\vdots$ & $\vdots$ & $\vdots$ & $\vdots$ & $\vdots$ \\
Opt $f_{m}(x, u)$ & $f_{1}\left(x_{m}^{*}, u_{m}^{*}\right)$ & $f_{2}\left(x_{m}^{*}, u_{m}^{*}\right)$ & $f_{3}\left(x_{m}^{*}, u_{m}^{*}\right)$ & $\cdots$ & $f_{m}\left(x_{m}^{*}, u_{m}^{*}\right)$ \\
\hline
\end{tabular}

For instance, $\left(x_{j}^{*}, u_{j}^{*}\right)$ represents the optimal solution of the $j$-th single objective optimization, then $f_{i}^{\max }$ can be achieved by

$$
f_{i}^{\max }=\max _{j=1, \cdots, m} f_{i}\left(x_{j}^{*}, u_{j}^{*}\right), \quad i=1, \cdots, m .
$$

The value of the membership function is also called satisfactory degree in this paper. 


\subsubsection{Relaxed order of satisfactory degrees}

Generally, preemptive priorities require the objectives be optimized in sequence. However, this may result in optimization of only a few objectives, not all the objectives, with a heavy computation burden.

Based on satisfactory optimization, preemptive priorities can be expressed by satisfactory degrees according to the principle that the objective with higher priority has higher satisfactory degree. For example, the requirement that $f_{t}(x, u)$ has a higher priority than $f_{s}(x, u)$ can be described by

$$
\mu_{f_{s}}(x, u) \leq \mu_{f_{t}}(x, u), \quad s, t \in\{1, \cdots, m\}, s \neq t .
$$

However, in some cases, (33) as a constraint is too strict to ensure the optimization problem feasible. In other cases, the satisfactory solution may not be acquired since some satisfactory degrees belonging to different priorities are equal. Therefore, it is necessary to introduce the parameter $\gamma$ to relax the order of satisfactory degrees (33), i.e.,

$$
\mu_{f_{s}}(x, u)-\mu_{f_{t}}(x, u) \leq \gamma, \quad s, t \in\{1, \cdots, m\}, s \neq t
$$

where $-1 \leq \gamma \leq 1$. When $\gamma \leq 0$, it means that the relaxed order of satisfactory degrees conforms to the basic preemptive priorities. On the contrary, the requirement of preemptive priorities cannot be guaranteed.

\subsubsection{FSGP model and algorithm}

For MOO, GP is a powerful tool. Its formulation is

$$
\begin{cases}\min & \sum_{i=1}^{k}\left(p_{i}+n_{i}\right) \\ \text { s.t. } & f_{i}(z)+n_{i}-p_{i}=f_{i}^{*}, \quad i=1, \cdots, k, \\ & n_{i}, p_{i} \geq 0, n_{i} \cdot p_{i}=0 \\ & z \in G\end{cases}
$$

where $p_{i}, n_{i}$ are the positive and negative deviational variable. According to (35), the objectives are transformed respectively in this paper.

Firstly, the three fuzzy relations " $\tilde{=}$ ", " $\tilde{\geq}$ " and " $\tilde{=}$ " are expressed into the new formulations using GP strategy.

For "文"

$$
f_{i}(x, u)-p_{i}=f_{i}^{*}, i=1, \cdots, m .
$$

Then its membership function (29) is rewritten as

$$
\mu_{f_{i}}(x, u)=1-\frac{p_{i}}{f_{i}^{\max }-f_{i}^{*}} .
$$

For " $\geq "$

$$
f_{i}(x, u)+n_{i}=f_{i}^{*}, \quad i=1, \cdots, m
$$

And

$$
\mu_{f_{i}}(x, u)=1-\frac{n_{i}}{f_{i}^{*}-f_{i}^{\min }} .
$$

For " $\tilde{=}$ ", we also have

$$
f_{i}(x, u)+n_{i}-p_{i}=f_{i}^{*}, \quad i=1, \cdots, m
$$

$$
\mu_{f_{i}}(x, u)=\left\{\begin{array}{l}
1-\frac{p_{i}}{f_{i}^{\max }-f_{i}^{*}} \\
1-\frac{n_{i}}{f_{i}^{*}-f_{i}^{\min }}
\end{array}\right.
$$

Suppose the case that the priority of $f_{j}(x, u)$ is higher than $f_{s}(x, u)$, and is lower than $f_{i}(x, u)$. Simultaneously, they have the following fuzzy relations

$$
\begin{array}{ll}
f_{i}(x, u) \tilde{\leq} f_{i}^{*}, & i=1, \cdots, m_{1} \\
f_{j}(x, u) \tilde{\geq} f_{j}^{*}, & j=m_{1}+1, \cdots, m_{2} \\
f_{s}(x, u) \tilde{=} f_{s}^{*}, & s=m_{2}+1, \cdots, m .
\end{array}
$$

Then, by substituting (37), (39), (40) into (34) according to the above priorities, the following relaxed orders of satisfactory degrees are constructed respectively to model preemptive priorities

$$
\begin{aligned}
& \frac{p_{i}}{f_{i}^{\max }-f_{i}^{*}}-\frac{n_{j}}{f_{j}^{*}-f_{j}^{\min }} \leq \gamma \\
& \frac{n_{j}}{f_{j}^{*}-f_{j}^{\min }}-\left[\frac{n_{s}}{f_{s}^{*}-f_{s}^{\min }}+\frac{p_{s}}{f_{s}^{\max }-f_{s}^{*}}\right] \leq \gamma .
\end{aligned}
$$

Combining (35)-(42), the generalized FSGP model can be formulated as

$$
\begin{aligned}
& \min \frac{1}{m}\left[\begin{array}{c}
\sum_{i=1}^{m_{1}} \frac{p_{i}}{f_{i}^{\max }-f_{i}^{*}}+ \\
\sum_{j=m_{1}+1}^{m_{2}} \frac{n_{j}}{f_{j}^{*}-f_{j}^{\min }}+ \\
\sum_{s=m_{2}+1}^{m}\left(\begin{array}{l}
\frac{n_{s}}{f_{s}^{*}-f_{s}^{\min }}+ \\
\frac{p_{s}}{f_{s}^{\max }-f_{s}^{*}}
\end{array}\right)
\end{array}\right]+\lambda \cdot \gamma \\
& \text { s.t. } \quad f_{i}(x, u)+n_{i}-p_{i}=f_{i}^{*}, i=1, \cdots, m_{1} \\
& f_{j}(x, u)+n_{j}-p_{j}=f_{j}^{*}, j=m_{1}+1, \cdots, m_{2} \\
& f_{s}(x, u)+n_{s}-p_{s}=f_{s}^{*}, s=m_{2}+1, \cdots, m \\
& \frac{p_{i}}{f_{i}^{\text {max }}-f_{i}^{*}}-\frac{n_{j}}{f_{j}^{*}-f_{j}^{\text {min }}} \leq \gamma \\
& \frac{n_{j}}{f_{j}^{*}-f_{j}^{\min }}-\left(\begin{array}{l}
\frac{n_{s}}{f_{s}^{*}-f_{s}^{\min }}+ \\
\frac{p_{s}}{f_{s}^{\max }-f_{s}^{*}}
\end{array}\right) \leq \gamma \\
& n_{j} \leq f_{j}^{*}-f_{j}^{\min }, p_{i} \leq f_{i}^{\max }-f_{i}^{*} \\
& n_{s} \leq f_{s}^{*}-f_{s}^{\min }, p_{s} \leq f_{s}^{\max }-f_{s}^{*} \\
& n_{i}, p_{i}, n_{j}, p_{j}, n_{s}, p_{s} \geq 0 \\
& n_{i} \cdot p_{i}=0, n_{j} \cdot p_{j}=0, n_{s} \cdot p_{s}=0 \\
& -1 \leq \gamma \leq 1,(x, u) \in G
\end{aligned}
$$

where the first part of the optimization objective of (43) is to optimize all the objectives by minimizing the deviations to aspiration values. The purpose of minimizing $\lambda \cdot \gamma$ is to maximize difference of priorities as far as possible. These two parts reflect the compromise between optimization and priorities. $\lambda$ is regulating parameter, it is equivalent to the weight of priorities. Its increase means the requirement of preemptive priorities will be strengthened. Otherwise, the minimization of deviations will be emphasized. That is, if $\gamma>0$, the priorities are not satisfied, then $\lambda$ needs to be increased till $\gamma \leq 0$ and the results are satisfactory. Therefore, how to balance optimization and priorities can be realized by changing $\lambda$. 
According to the proposed FSGP method, the following algorithm is given as follows:

Step 1. Formulate the optimization model (43) based on the type of fuzzy relations and preemptive priorities of the objectives of the reentry trajectory optimization problem.

Step 2. Determine initial value of $\lambda$ and solve the optimization model established in Step 1.

Step 3. If $\gamma>0$, the priorities are not satisfied, go to Step 4. If $\gamma \leq 0$, determine the satisfaction of solutions: when the current solution is satisfactory, optimization stops; otherwise, go to the next step.

Step 4. Increase $\lambda$ and solve the optimization model again, then go back to Step 3 and continue.

\subsection{Analysis of optimization method}

From the proposed FSGP algorithm, we know that the satisfactory result can be acquired by regulating the parameter $\lambda$. Thus, iteration may happen during solving (43), which increases the computation of the algorithm. However, it can be concluded that the number of iterations is limited. According to $\mathrm{Hu}^{[20]}$, there must exists $\lambda^{*}$. When $\lambda>\lambda^{*}$, the solution of (43) $\gamma$ will remain unchanged. Then iteration stops. Therefore, the computation burden depending on iteration is not heavy. $\lambda^{*}$ can be determined by combining model (43) with Kuhn-Tucker (K-T) condition. Firstly, the maximum difference of priorities $\gamma^{*}$ is obtained by the following model

$$
\left\{\begin{array}{cc}
\min & \gamma \\
\text { s.t. } & \text { constraints of (43). }
\end{array}\right.
$$

Then, combining $\gamma^{*}$ and the K-T condition, the Lagrange function $F(x, u, n, p, \gamma)$ is constructed and differentiated. Finally, $\lambda^{*}$ can be acquired through solving (45).

$$
\left\{\begin{array}{cc}
\min & \lambda \\
\text { s.t. } & \frac{\partial F}{\partial(x, u, n, p, \gamma)}=0 \\
& \text { Lagrange multipliers equations } \\
& \lambda \geq 0 .
\end{array}\right.
$$

Considering the whole reentry trajectory approach, the optimization procedure can be summarized as follows:

1) Formulate the discrete nonlinear multi-objective programming problem with preemptive priorities using direct collocation method.

2) Solve the multi-objective programming problem by FSGP algorithm to acquire the satisfactory reentry trajectory.

\section{Simulation}

\subsection{Parameters of simulation}

The mass and reference area of the vehicle are given as $m=7008$ slugs and $S=2690 \mathrm{ft}^{2}$; the maximum values of heat flux, dynamic pressure and overload are $\dot{Q}_{\max }=$ $200 \mathrm{Btu} / \mathrm{ft}^{2} / \mathrm{s}, q_{\max }=280 \mathrm{lb} / \mathrm{ft}^{2}$ and $n_{\max }=2.5$; the initial and terminal data of reentry process and boundary constraints of state and control variables are shown in Table 2.

Table 2 Initial and terminal constraints and boundary constraints

\begin{tabular}{ccccc}
\hline & Initial & Terminal & Minimum & Maximum \\
\hline$r(\mathrm{ft})$ & 21162900 & 20982900 & 20902900 & 21202900 \\
$\theta(\mathrm{deg})$ & 0 & & -90 & 90 \\
$\phi(\mathrm{deg})$ & 0 & & -89 & 89 \\
$v(\mathrm{ft} / \mathrm{s})$ & 25600 & $\geq 2000$ & 1 & 30000 \\
$\chi(\mathrm{deg})$ & 90 & & -180 & 180 \\
$\gamma(\mathrm{deg})$ & -1 & -5 & -89 & 89 \\
$\alpha(\mathrm{deg})$ & 17.42 & & -10 & 30 \\
$\sigma(\mathrm{deg})$ & -75 & & -80 & 80 \\
\hline
\end{tabular}

Set the whole reentry flight time as $2010 \mathrm{~s}$, and divide the whole time into 20 segments, so that 21 nodes are selected including the initial points.

\subsection{Results and analysis}

Firstly, we get the optimization result of single objective reentry trajectory according to the motion equations, objectives and constraints given in Section 2. The results are shown in Figs. 1-4.

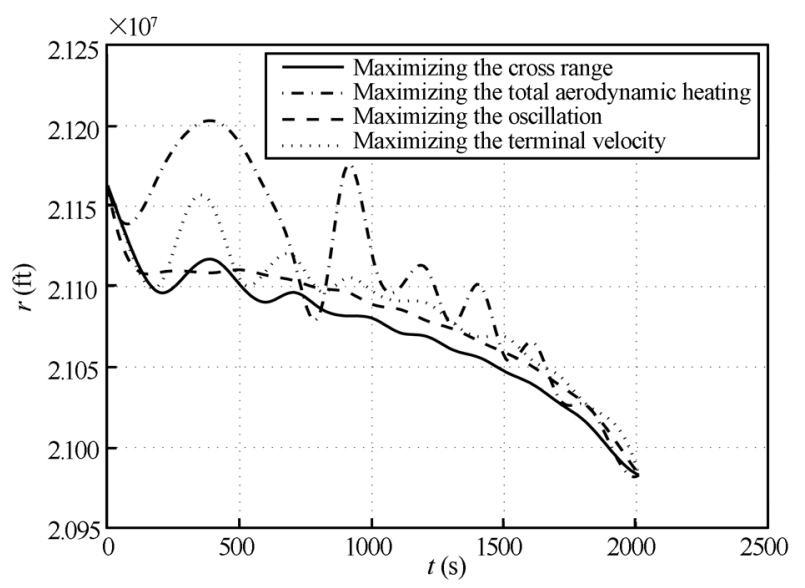

Fig. 1 Radial position profile of single objective

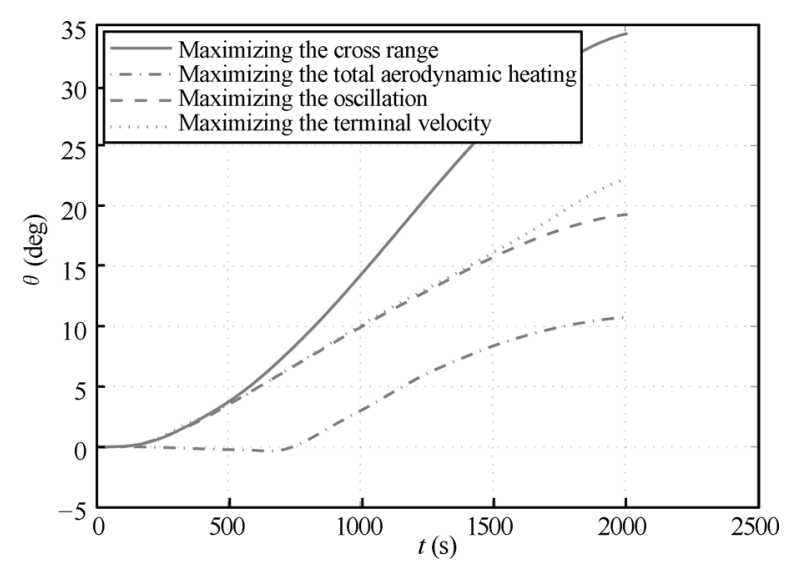

Fig. 2 Longitude profile of single objective 


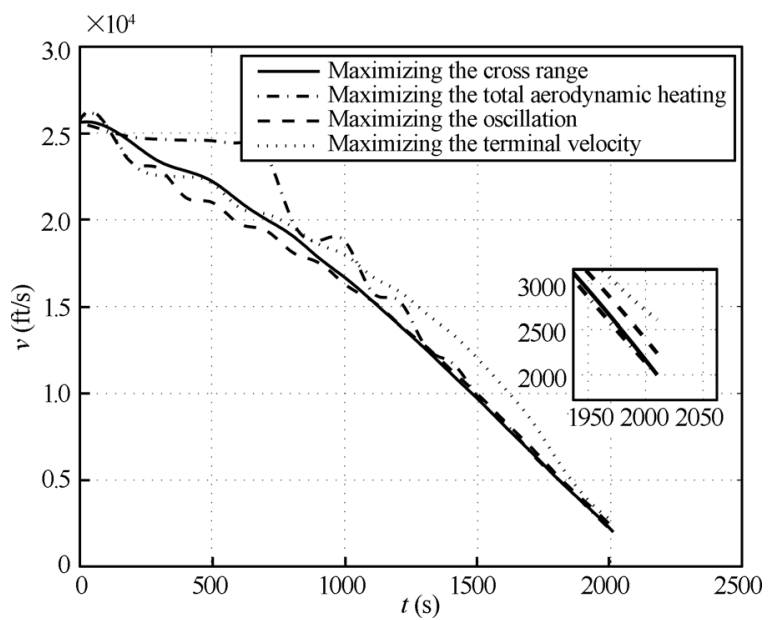

Fig. 3 Velocity profile of single objective

From Fig. 1 to Fig. 4, we can see that the optimal values for different objectives are not consistent. This indicates that conflicts exist among objectives. So it is impossible to find a solution optimizing each objective.

Thus, the proposed reentry trajectory optimization method based on FSGP is adopted.

Firstly, determine the perspective goal value and the lower and upper tolerance limits of each objective by the above optimization results of single objective cases. The payoff table is established as Table 3 .

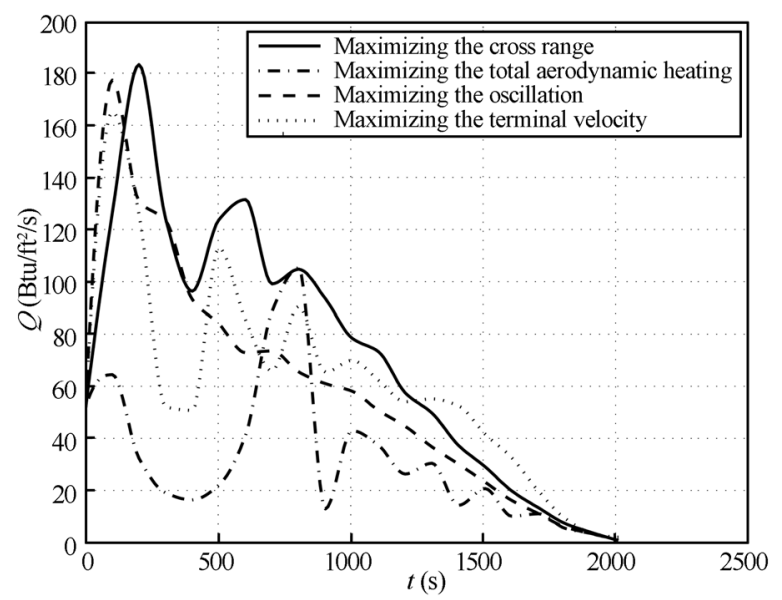

Fig. 4 Heat flux profile of single objective

Table 3 Payoff table of reentry trajectory with $\mathrm{MOO}$

\begin{tabular}{ccccc}
\hline & $f_{1}$ & $f_{2}$ & $f_{3}$ & $f_{4}$ \\
\hline $\max f_{1}(x, u)$ & 0.5989 & 184.4798 & 0.3877 & 0.0771 \\
$\min f_{2}(x, u)$ & 0.1881 & 78.7396 & 1.6598 & 0.0771 \\
$\min f_{3}(x, u)$ & 0.3365 & 148.2756 & $1.0311 \times 10^{-7}$ & 0.0863 \\
$\max f_{4}(x, u)$ & 0.3889 & 154.9605 & 1.8119 & 0.1004 \\
\hline
\end{tabular}

From payoff table, it is concluded that

$$
\begin{array}{ll}
f_{1}^{*}=0.5989 & f_{1}^{\min }=0.1881 \\
f_{2}^{*}=78.7396 & f_{2}^{\max }=184.4798 \\
f_{3}^{*}=1.0311 \times 10^{-7} & f_{3}^{\max }=1.8119 \\
f_{4}^{*}=0.1004 & f_{4}^{\min }=0.0771 .
\end{array}
$$

Then, the following FSGP model as (43) is established with the given preemptive priorities

$$
\begin{cases}\min & \frac{1}{4}\left(\begin{array}{l}
\frac{n_{1}}{0.5989-0.1881}+p_{2} \\
\frac{p_{3}}{184.479-78.7396}+ \\
\frac{p_{3}}{1.819-0}+ \\
\frac{n_{4}}{0.1004-0.0771}
\end{array}\right)+\lambda \cdot \gamma \\
\text { s.t. } & \theta\left(t_{f}\right)+n_{1}-p_{1}=0.5989 \\
& \int_{t_{0}}^{t_{f}} \dot{Q}(t) \mathrm{d} t+n_{2}-p_{2}=78.7396 \\
& \int_{t_{0}}^{t_{f}} \dot{\gamma}^{2}(t) \mathrm{d} t+n_{3}-p_{3}=1.0311 \times 10^{-7} \\
& u\left(t_{f}\right)+n_{4}-p_{4}=0.1004 \\
& \frac{n_{1}}{0.5989-0.1881}-\frac{p_{2}}{184.4798-78.7396} \leq \gamma \\
& \frac{n_{4}}{0.1004-0.0771}-\frac{p_{2}}{184.4798-78.7396} \leq \gamma \\
& \frac{p_{2}}{184.4798-78.7396}-\frac{p_{3}}{1.8119-0} \leq \gamma \\
& n_{1} \leq(0.5989-0.1881), p_{2} \leq(184.4798-78.7396) \\
& p_{3} \leq(1.8119-0), n_{4} \leq(0.1004-0.0771) \\
& n_{1} \cdot p_{1}=0, n_{2} \cdot p_{2}=0, n_{3} \cdot p_{3}=0, n_{4} \cdot p_{4}=0 \\
& n_{1}, p_{1}, n_{2}, p_{2}, n_{3}, p_{3}, n_{4}, p_{4} \geq 0,-1 \leq \gamma \leq 1 \\
& \dot{Q}=\left(\begin{array}{c}
h_{0}+h_{1} \alpha+ \\
h_{2} \alpha^{2}+h_{3} \alpha^{3}
\end{array}\right) \cdot C \rho^{0.5} v^{3.07} \leq 200 \mathrm{Btu} / \mathrm{ft}^{2} / \mathrm{s} \\
& \frac{\rho v^{2}}{2} \leq 280 \mathrm{lb} / \mathrm{ft}^{2} \\
& \sqrt{L^{2}+D^{2}} \leq 2.5 \mathrm{mg} \\
& (11),(12),(16),(17) \text { combining with Table } 2 .\end{cases}
$$

Regulating $\lambda$ and solving the above model, different $\gamma$ and satisfactory degrees of the objectives are achieved. They are listed in Table 4.

Table 4 Optimization results for different $\lambda$

\begin{tabular}{ccc}
\hline$\lambda$ & $\gamma$ & $\begin{array}{c}\text { Satisfactory degrees of objectives } \\
\left(\mu_{f_{1}}, \mu_{f_{2}}, \mu_{f_{3}}, \mu_{f_{4}}\right)\end{array}$ \\
\hline 0.35 & 0.1009 & $(0.7081,0.8090,0.9098,1.0000)$ \\
0.4 & 0.0698 & $(0.7234,0.7932,0.8630,1.0000)$ \\
0.45 & -0.0353 & $(0.7687,0.7334,0.6981,1.0000)$ \\
0.5 & -0.2170 & $(0.8331,0.6161,0.3991,1.0000)$ \\
\hline
\end{tabular}

From Table 4, it can be concluded that with the change of parameter $\lambda$, the satisfactory degree of each objective changes along the direction of priority. When $\lambda=0.35$ and $\lambda=0.4, \gamma>0$, and the order of priorities is not satisfied according to satisfactory degrees. With the increase of $\lambda$, the satisfactory degree of the objective a) is raising. But the satisfactory degrees of the objective b) and the objective c) are decreasing gradually. The declining rate of the latter is higher than that of the former because the objective $b$ ) has the higher priority than the objective $\mathrm{c}$ ). When $\lambda=$ 0.5 , the satisfactory degree of the objective c) has declined to 0.3991 , denoting that this objective is optimized badly. On the other hand, the objective a) has obtained a better result, which proves the fact that there exist conflicts among the objectives. In particular, the satisfactory degree of the objective d) maintains 1.000 for all $\lambda$. This denotes that this objective can always be optimized enough using our approach.

The corresponding trajectories for different $\lambda$ are shown in Figs. 5-15. 


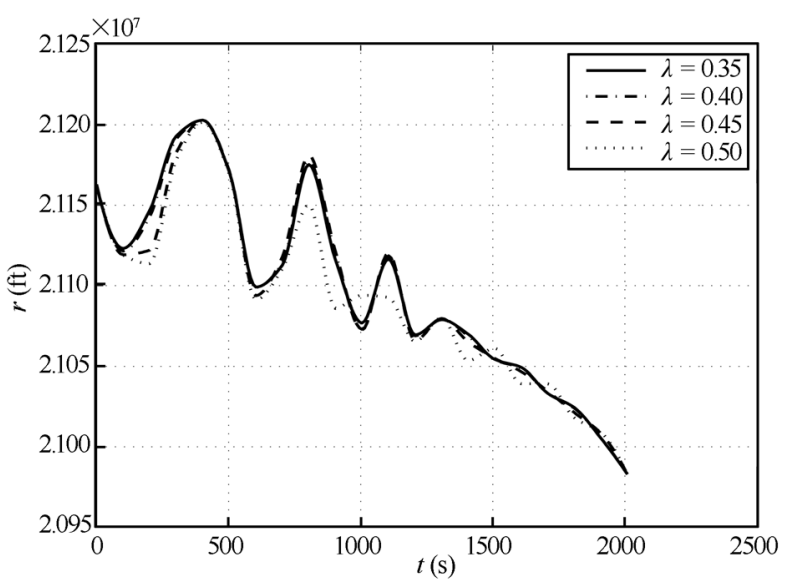

Fig. 5 Radial position profile with different $\lambda$

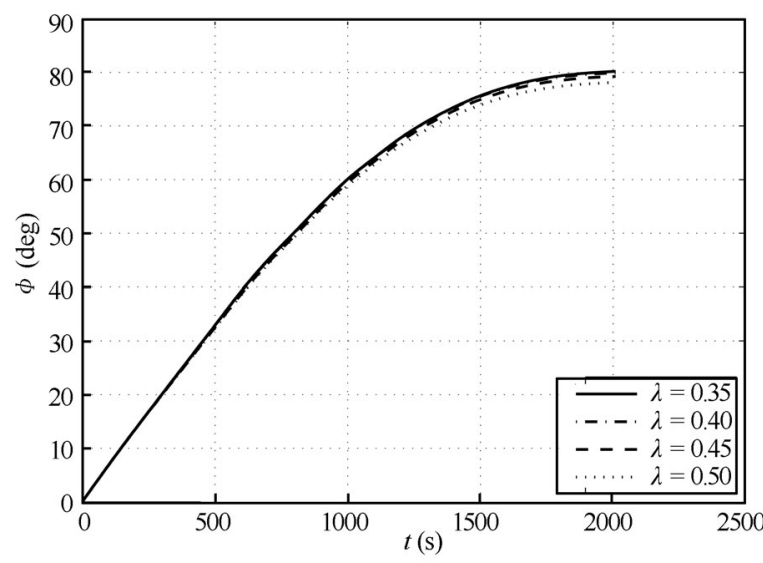

Fig. 6 Latitude profile with different $\lambda$

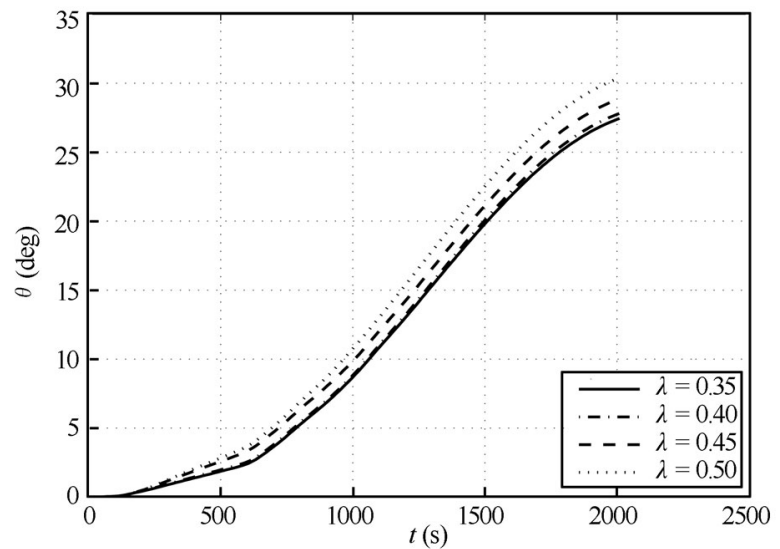

Fig. 7 Longitude profile with different $\lambda$

Latitude and longitude shown in Figs. 6 and 7 increase smoothly, and terminal longitude reflects the changes of the objective a), i.e., it grows with the increase of $\lambda$. Velocity and heading angle shown in Figs. 8 and 9 decrease smoothly, and terminal velocity is keeping around $2600 \mathrm{ft} / \mathrm{s}$. Flightpath angle shown in Fig. 10 maintains the trend of smaller oscillation when $\lambda$ decreases. Shown as Fig. 5, the oscilla- tion amplitude of radial position attenuates gradually. Attack angle shown in Fig. 11 maintains greater values in the early reentry, which can reduce the aerodynamic heating and help stabilize the trajectory. But it decreases in the later phase to achieve a larger range. Bank angle profile shown in Fig. 12 has a large vibration in the early stage and tends to zero gradually. The path constraints shown in Figs. $13-15$ are all limited in the maximum permissible values.

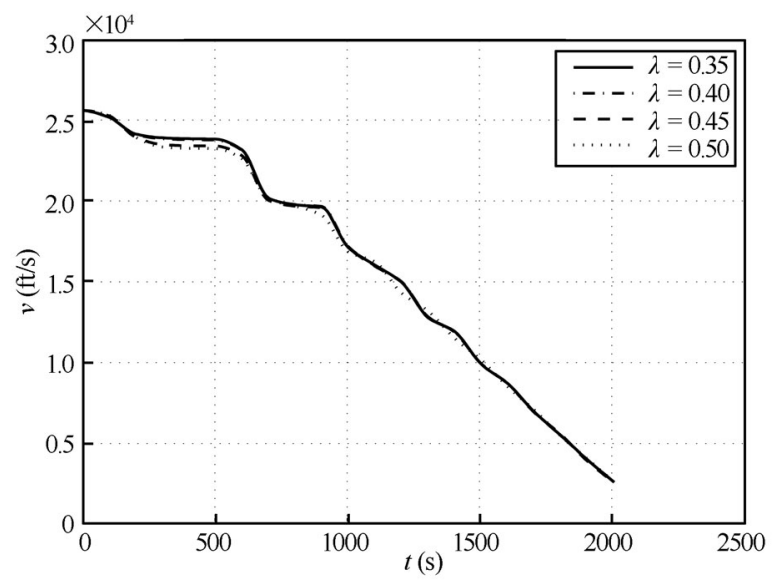

Fig. 8 Velocity profile with different $\lambda$

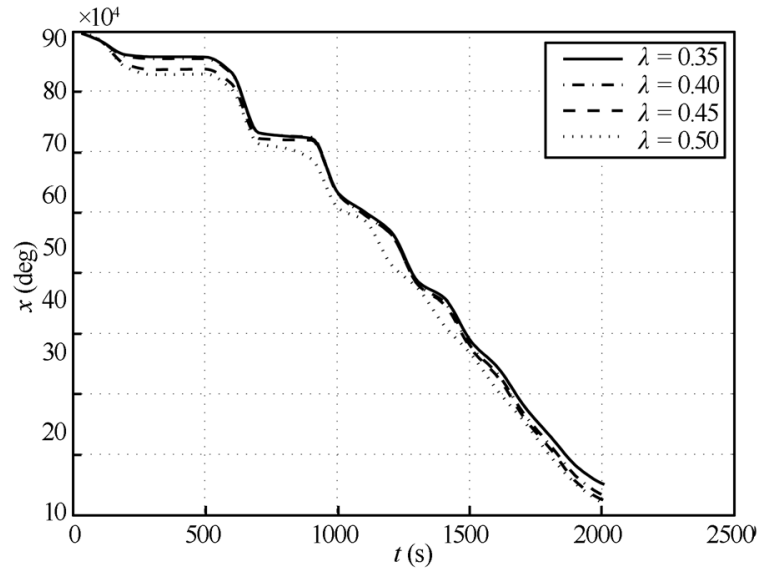

Fig. 9 Heading angle profile with different $\lambda$

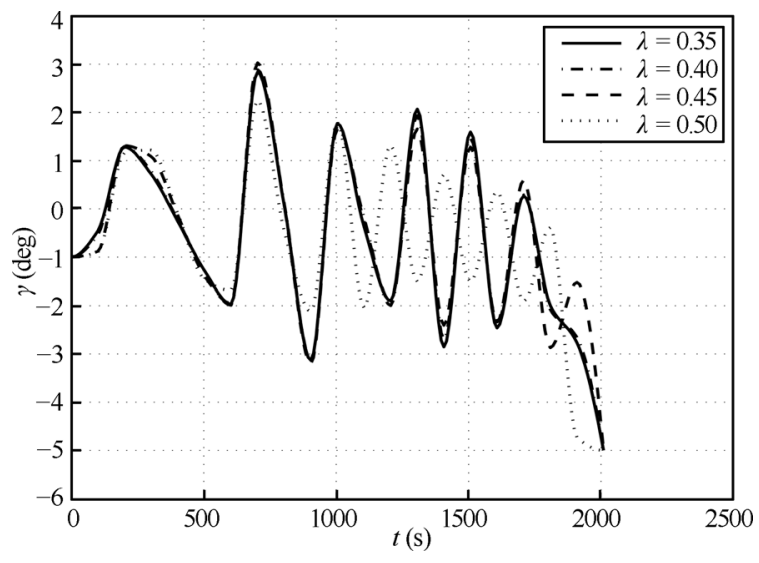

Fig. 10 Flight-path angle profile with different $\lambda$ 


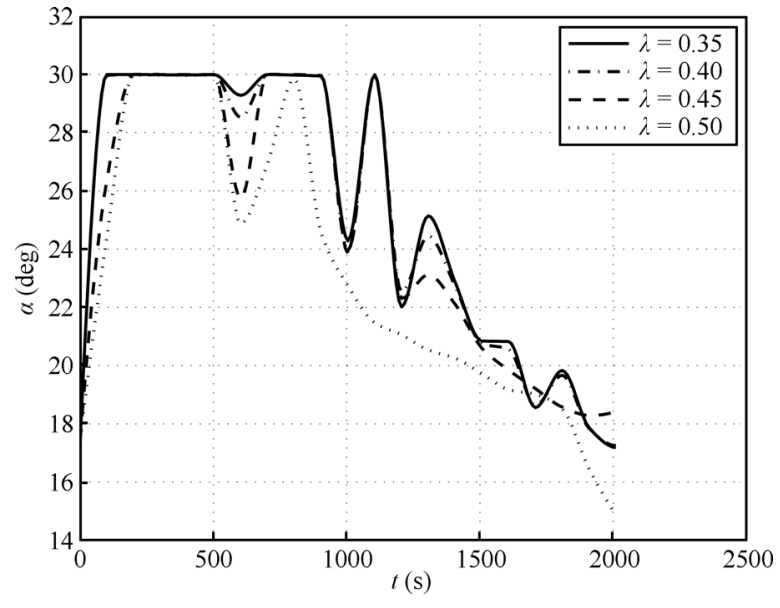

Fig. 11 Attack angle profile with different $\lambda$

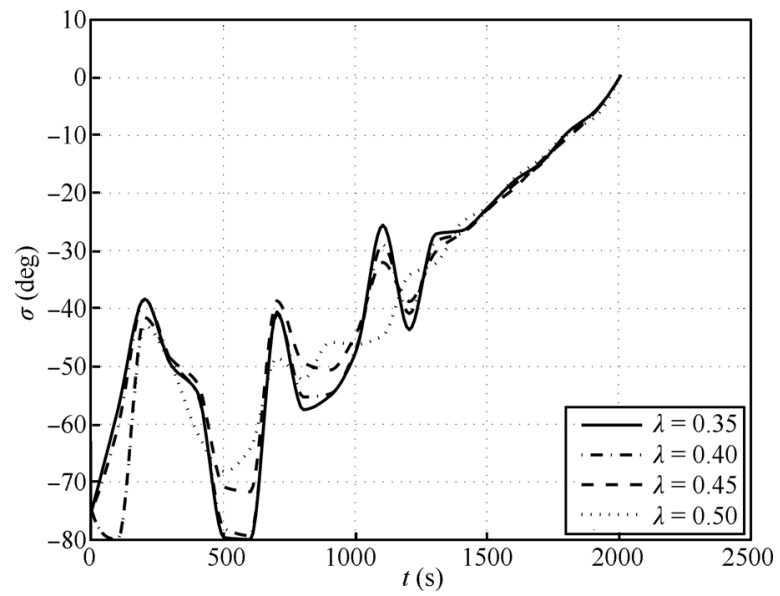

Fig. 12 Bank angle profile with different $\lambda$

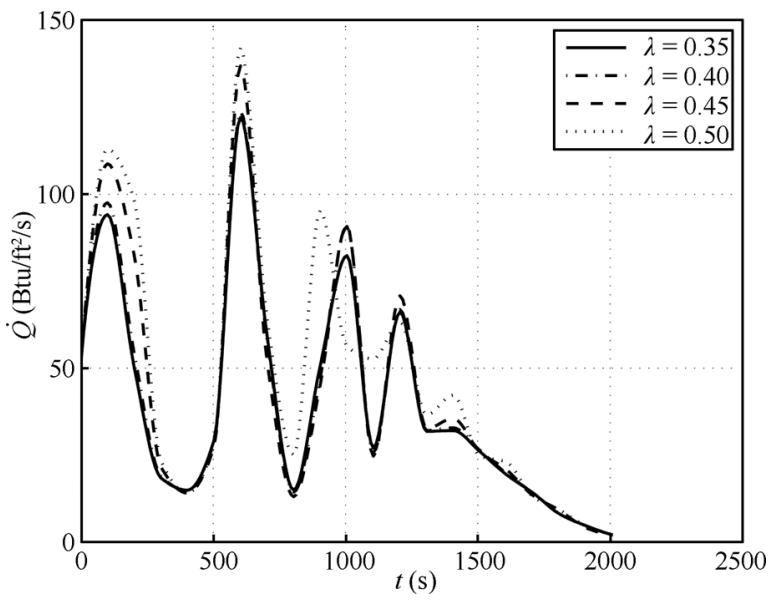

Fig. 13 Heat flux profile with different $\lambda$

From the above results, it is known that when $\lambda=0.45$, not only preemptive priorities among the objectives are proper, but also the objectives of maximum cross range and maximum terminal velocity are optimized enough. This satisfies the actual requirement. Therefore, the corresponding trajectory with $\lambda=0.45$ is selected as the optimal trajectory.

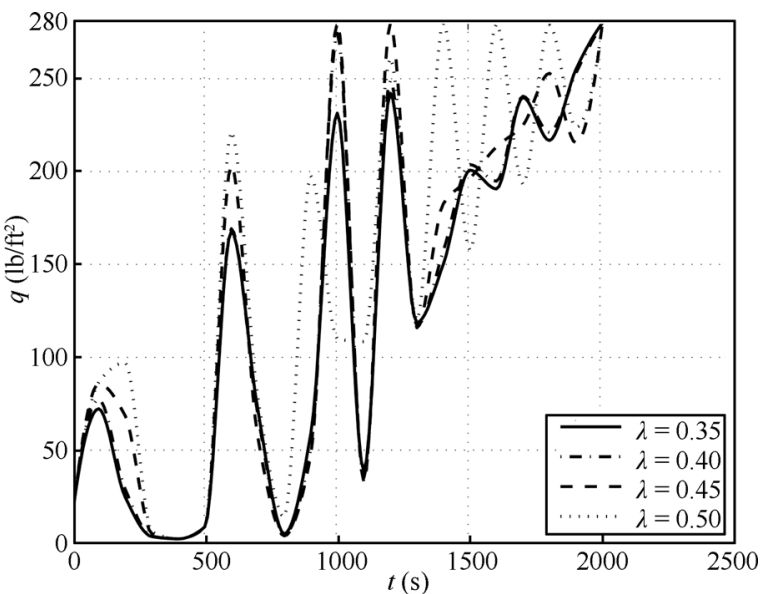

Fig. 14 Dynamic pressure profile with different $\lambda$

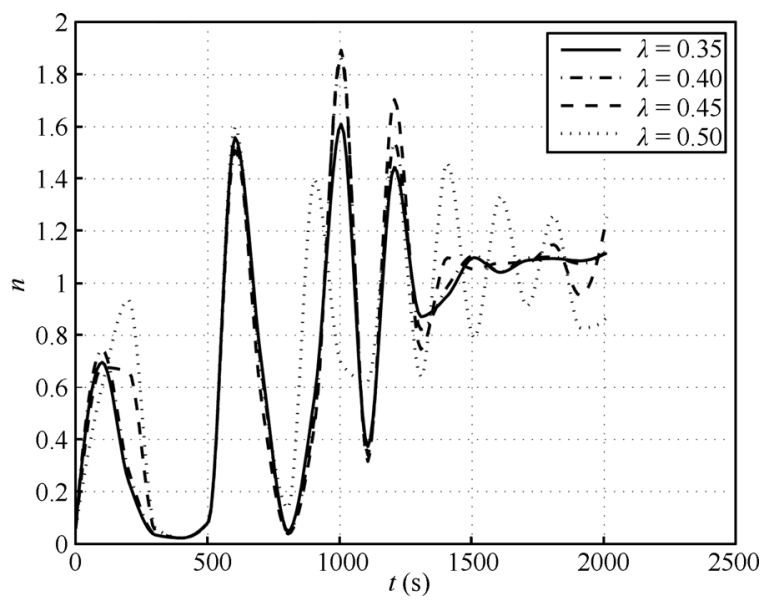

Fig. 15 Overload profile with different $\lambda$

For validating the effectiveness of the proposed method, the traditional weighted approach is adopted to solve the reentry trajectory with $\mathrm{MOO}$ problem. By selecting some different combinations of weights, we can get different optimization results, listed in Table 5 .

From Table 5, we can know that even though the weights are given according to the priorities, the satisfactory degrees do not fully conform to the priorities. For example, in the first case, the satisfactory degree of the objective a) is lower than that of the objective c). In the second and the fourth case, the satisfactory degree of the objective c) is higher than b), which also destroys the priorities. Although the result of the third case satisfies the order of preemptive priorities, the solution is inferior and it is not taken as the optimization solution of MOO.

Table 5 Optimization results for weighted method

\begin{tabular}{cc}
\hline Weights & $\begin{array}{c}\text { Satisfactory degrees of objectives } \\
\left(\mu_{f_{1}}, \mu_{f_{2}}, \mu_{f_{3}}, \mu_{f_{4}}\right)\end{array}$ \\
\hline$(0.3,0.25,0.15,0.3)$ & $(0.7898,0.6638,0.9686,1.0000)$ \\
$(0.35,0.2,0.1,0.35)$ & $(0.5260,0.3463,0.4303,0.8324)$ \\
$(0.4,0.15,0.05,0.4)$ & $(0.5059,0.3640,0.0685,0.8574)$ \\
$(0.45,0.099,0.001,0.45)$ & $(0.9592,0.2587,0.9083,1.0000)$ \\
\hline
\end{tabular}


Compared with the method based on weighting factors, the FSGP method has its advantages. Firstly, the priorities can be satisfied strictly in the FSGP approach when $\gamma \leq 0$, however, the weighted one cannot ensure this. Secondly, in the FSGP method, different satisfactory degrees of the objectives are obtained by adjusting the parameter, it is much simpler than the determination of multiple weights. Thirdly, the inferior solution does not exist for the FSGP method. Nevertheless, it is not guaranteed using weighting factors.

Remark 1. In multi-objective reentry trajectory optimization problem, attack angle may approach its boundary because it can reduce aerodynamic heating of the vehicle especially when the total aerodynamic heating is considered as the optimization objective. Simultaneously, the boundary of attack angle can be taken as soft constraint in the reformulated MOO model. Thus, its boundary can be regulated in order to avoid the infeasibility resulted from high attack angle. The optimization result is still suitable for reentry trajectory optimization.

\section{Conclusions}

In this work, the reentry trajectory design problem of hypersonic vehicles with multiple objectives and constraints is solved by FSGP method. The original continuous trajectory optimization problem is transformed into discrete static nonlinear programming problem by direct collocation method. For the preemptive priorities among objectives, the relaxed order of satisfactory degrees is constructed. Based on this, the fuzzy satisfactory goal programming model is formulated to compromise the conflicts among the objectives and balance optimization and priorities. This method can not only avoid strong nonlinearities and complex calculations in traditional MOO methods, but also obtain the satisfactory reentry trajectory meeting the requirements of optimization and priorities.

\section{Acknowledgments}

The authors would like to thank the anonymous reviewers for their helpful comments and constructive suggestions with regard to this paper.

\section{References}

[1] T. J. Betts. Survey of numerical methods for trajectory optimization. Journal of Guidance, Control, and Dynamics, vol. 21, no. 2, pp. 193-207, 1998.

[2] E. M. Yong, L. Chen, G. J. Tang. A survey of numerical methods for trajectory optimization of spacecraft. Journal of Astronautics, vol. 29, no. 2, pp. 397-406, 2008.

[3] O. V. Stryk, R. Bulirsch. Direct and indirect methods for trajectory optimization. Annals of Operations Research, vol. 37, no. 1, pp. 357-373, 1992.
[4] X. J. Qu. The application of the multiple shooting algorithm to trajectory optimization. Flight Dynamics, vol. 10, no. 1, pp. 13-21, 1992.

[5] A. B. David, T. H. Geoffrey, A. V. Rao. Direct trajectory optimization and costate estimation via an orthogonal collocation method. Journal of Guidance, Control, and Dynamics, vol. 29, no. 6, pp. 1435-1440, 2006.

[6] L. H. Albert, A. C. Bruce. Direct optimization using collocation based on high-order Gauss-Lobatto quadrature rules. Journal of Guidance, Control and Dynamics, vol. 19, no. 3 , pp. 592-599, 1996.

[7] G. T. Huntington, A. V. Rao. Optimal reconfiguration of spacecraft formations using the Gauss pseudospectral method. Journal of Guidance, Control, and Dynamics, vol. 31, no. 3, pp. 689-698, 2008.

[8] R. T. Bibeau. Trajectory Optimization for a Fixed Trim Re-entry Vehicle Using Direct Collocation with Nonlinear Programming, Ph. D. dissertation, Massachusetts Institute of Technology, USA, 1999.

[9] L. H. Tu, J. P. Yuan. Reentry skipping trajectory optimization using direct parameter optimization method. In Proceedings of the 14th AIAA/AHI International Space Planes and Hypersonic Systems Technologies Conference, AIAA, Canberra, Australia, pp. 824-837, 2006.

[10] G. Chen, Y. Hu, M. Xu, Z. M. Wan, S. L. Chen. Implementing a multi-objective optimization design of RLV reentry trajectory. Journal of Northwestern Polytechnical University, vol. 24, no. 2, pp. 133-137, 2006.

[11] E. M. Yong, L. Chen, G. J. Tang. Trajectory optimization of hypersonic gliding reentry vehicle based on the physical programming. Acta Aeronautica et Astronautica Sinica, vol. 29, no. 5, pp. 1091-1097, 2008.

[12] B. B. Pal, B. N. Moitra. A goal programming procedure for solving problems with multiple fuzzy goals using dynamic programming. European Journal of Operation Research, vol. 144, no. 3, pp. 480-491, 2003.

[13] S. Y. Li, Y. P. Yang. Fuzzy goal programming with multiple priorities via generalized varying-domain optimization method. IEEE Transactions on Fuzzy Systems, vol. 12, no. 5, pp. 596-605, 2004.

[14] S. Y. Li, C. F. Hu. Two-step interactive satisfactory method for fuzzy multiple objective optimization with preemptive priorities. IEEE Transactions on Fuzzy Systems, vol. 15, no. 3, pp. 417-425, 2007.

[15] A. Charnes, W. W. Cooper. Management models and industrial applications of linear programming. Management Science, vol. 4, no. 1, pp. 38-91, 1957.

[16] L. H. Chen, F. C. Tsai. Fuzzy goal programming with different importance and priorities. European Journal of Operational Research, vol. 133, no. 3. pp. 548-560, 2001. 
[17] B. L. Tian, Q. Zong. Optimal guidance for reentry vehicles based on indirect Legendre pseudospectral method. Acta Astronautica, vol. 68, no. 7-8, pp. 1176-1184, 2011.

[18] L. H. Tu, J. P. Yuan, X. K. Yue, J. J. Luo. Improving design of reentry vehicle trajectory optimization using direct collocation method. Journal of Northwestern Polytechnical University, vol. 24, no. 5, pp. 654-657, 2006.

[19] C. F. Hu, S. Y. Li. Enhanced interactive satisfying optimization approach to multiple objective optimization with preemptive priorities. International Journal of Information Technology \& Decision Making, vol. 5, no. 1, pp.47-63, 2006 .

[20] C. F. Hu. Research on Fuzzy Multiple Objective Optimization Based on Decision Maker's Satisfying Degree, Ph. D. dissertation, Shanghai Jiao Tong University, China, 2007.

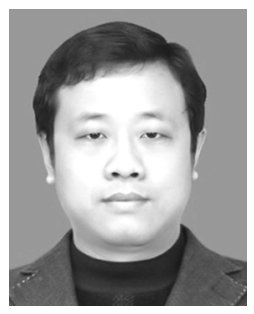

author)
Chao-Fang $\mathbf{H u}$ received his $\mathrm{Ph}$. D. degree from Shanghai Jiao Tong University, China in 2007. He is currently an associate professor at School of Electrical Engineering and Automation, Tianjin University.

His research interests include aircraft control, adaptive control, multi-objective optimization and fuzzy modeling.

E-mail: cfhu@tju.edu.cn (Corresponding ORCID iD: 0000-0002-3461-302X

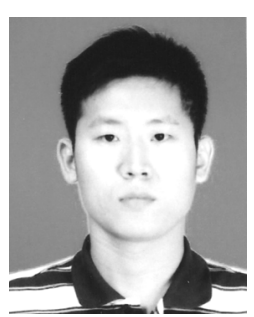

Yue Xin obtained his B. Sc. degree from Central South University, China in 2012. He is currently a graduate student at School of Electrical Engineering and Automation, Tianjin University.

His research interests include trajectory optimization and guidance of aircraft.

E-mail: xinyue_tju@163.com 